\title{
Processing Negation Without Context - Why and When We Represent the Positive Argument
}

\author{
Ye Tian ${ }^{1}$, Heather Ferguson ${ }^{2}$, and Richard Breheny ${ }^{3}$ \\ ${ }^{1}$ Laboratorie de Linguistique Formelle, Université Paris Diderot, France. \\ Postal Address : Salle 751, Olympe de Gouges, Rue Albert Einstein, 75013, Paris, \\ France. Telephone Number : (+33)0782896605. Email: tiany.03@gmail.com \\ ${ }^{2}$ School of Psychology, University of Kent, UK.
}

Postal Address: University of Kent, Canterbury, Kent, CT2, 7NP. Telephone Number: +44 (0)1227 827120. Email: H.Ferguson@kent.ac.uk

${ }^{3}$ Division of Psychology and Language Sciences, University College London, UK.

Postal Address: Room 108, Chandler House, UCL, Wakefield Street, London. Telephone Number: +44 (0)20 7679 4039. Email: r.breheny@ucl.ac.uk

Corresponding author: Ye Tian.

This work was supported by the Centre for the Study of Mind in Nature (Oslo), "Linguistic Agency" project. 


\begin{abstract}
When processing negative sentences without context, participants often represent states of the positive arguments. Why and when does this occur? Using visual world eyetracking, participants listened to positive and negative sentences in simple or cleft forms (e.g. [It is] Matt [who] hasn’t shut his dad's window), while looking at scenes containing a target and a competitor (matches or mismatches the implied shape of the final noun). Results show that in the simple but not the cleft condition, there is a difference between negatives and positives: shortly after the verb, there is more looks to the competitor in the simple negatives than the positives. This suggests that the representation of the positive is not a mandatory first step of negation processing (as per rejection accounts). Rather results support the Question Under Discussion (QUD) accommodation account wherein both sentence content and contextual source of relevance are targets of incremental sentence processing.
\end{abstract}

Keywords: negation; Question Under Discussion, pragmatics, visual world 
In research on negative sentence processing, across a range of different paradigms, results very often point to the conclusion that participants represent the positive argument of negation while performing reading or verification tasks, especially in the early stages of processing. For a sentence like, "The banana is not peeled", the positive argument of negation would be that the banana is peeled ${ }^{1}$. Specifically, responses in sentence verification tasks (Clark \& Chase, 1972; Carpenter \& Just, 1975; Dale \& Duran, 2011), probe recognition tasks (Kaup, Yaxley, Madden, Zwaan, \& Lüdtke, 2007; Lüdtke \& Kaup, 2006, (Hasson \& Glucksberg, 2006) and ERP studies (Fischler, Bloom, Childers, Roucos, \& Perry, 1983; Lüdtke, Friedrich, De Filippis, \& Kaup, 2008) strongly suggest that a representation of the positive argument of negation is employed in the process.

In sentence verification research, many studies show a polarity by truth-value interaction in response times. Clark and Chase (1972) asked participants to verify affirmative or negative sentences against pictures. For example, against a picture of a plus above a star, the sentences come in four conditions:

TA (True Affirmative): The plus is above the star.

FA (False Affirmative): The star is above the plus.

TN (True Negative): The star isn't above the plus.

FN (False Negative) : The plus isn't above the star.

They found that while for affirmative sentences, true sentence-picture pairs are faster than false sentence-picture pairs $(\mathrm{TA}<\mathrm{FA})$, the opposite is true for negative sentences $(\mathrm{TN}>\mathrm{FN})$. This pattern is interpreted in terms of a strategy based on the truth-functional property of negation: a negative sentence has the opposite truth-value of its positive counterpart. When presented with a negative sentence, participants first represent and evaluate the truth value of its positive argument (the corresponding 
affirmative), and then reverse the truth value. Given that response latencies are greater for FAs than TAs, it explains why TNs have longer response latencies than FNs. Dale and Duran (2011, experiment 1) conducted a sentence-world knowledge verification study using mouse-tracking ("Elephants are not small/large") and found a similar pattern.

Beyond sentence verification, in a series of studies using visual probe recognition, Kaup and colleagues established that at a short latency (250ms), participants are faster to respond to an image consistent with the positive argument of a negative sentence than an image consistent with the negative sentence itself (Kaup, Zwaan, \& Lüdtke, 2007). Thus, having read, "The bird was not in the air", participants responded faster to an image of a flying bird than one of a bird at rest. At longer latencies (1500ms), the pattern has been found to be reversed (Kaup, Zwaan, \& Lüdtke, 2006). In ERP studies, Fischler et al. (1983) found that certain kinds of TNs (e.g. "A robin is not a tree") give rise to greater N400 effects than corresponding FNs (e.g. "A robin is not a bird"), while the corresponding FAs predictably give rise to an N400 effect relative to TAs. Fischler et al. (1983) attribute this reversal of the normal N400 effect to the fact that participants first process the positive argument of negation. Lüdtke et al. (2008) measured ERPs when participants had to verify sentences such as "In the front of the tower there is a/no ghost" against a matching or mismatching picture presented $250 \mathrm{~ms}$ or $1500 \mathrm{~ms}$ after the sentence. They found that at $250 \mathrm{~ms}$ ISI, pictures that match the positive argument (e.g. a ghost in front of a tower) are primed equally by both positive and negative sentences. However, at 1500ms, pictures that match the positive argument were less primed than pictures that were consistent with the sentence meaning in the negative condition. They concluded that a negative sentence is processed by first simulating the positive argument. Negation is only integrated into the sentence 
meaning at a later point. Hasson and Glucksberg (2006) studied the processing of negative metaphors. They found that $150 \mathrm{~ms}$ or $500 \mathrm{~ms}$ after reading a negative sentence like "this lawyer is not a shark", participants were faster at making a lexical decision on a probe related to the positive counterpart (vicious) than one that is related to the negative sentence meaning (gentle). At $1000 \mathrm{~ms}$, the pattern is reversed. They conclude that negations are initially represented as their positive counterpart, and it takes between $500 \mathrm{~ms}$ and $1000 \mathrm{~ms}$ to arrive at the negation-consistent meaning.

While it seems that participants do sometimes represent the positive argument during various tasks in these studies, many studies suggest that they do not always. In the sentence verification literature, a second commonly reported pattern of results is a main effect of both polarity and truth-value, i.e. for both affirmative and negative sentences, true sentence-picture pairs are faster to verify than false sentence-picture pairs (Arroyo, 1982; Trabasso \& Rollins, 1971). This pattern is widely interpreted as being due to participants inferring what would make the negative sentence true (e.g. the situation of an open door for "The door is not closed") and checking that the image is consistent with this. Thus true sentences are verified faster, regardless of their polarity. In the ERP literature, Nieuwland and Kuperberg (2008) show that contextually felicitous TNs (e.g. “With proper equipment, scuba-diving isn’t very dangerous and often good fun.") do not give rise to an N400 effect compared to either TAs ("With proper equipment, scuba-diving is very safe and often good fun.") or FNs ("With proper equipment, scuba-diving isn't very safe and often good fun.”). Similarly, Dale and Duran (2011, Experiment 2\&3) indicate that the more contextual support the negative sentences have, the less the tendency there is to consider the positive argument. Several fMRI studies on negation (Tettamanti et al., 2008; Tomasino, Weiss, \& Fink, 2010) found no evidence of the representation of the positive argument during negative 
sentence processing. Tettamanti et al. (2008) show that while reading a positive sentence with action verbs (e.g. "grip", "clasp") activates the motor brain regions, negation modulates this activity. Specifically, negative phrases tend to show decreased activation relative to their positive counterparts. Finally, following on from Kaup et al. (2007), Tian, Breheny and Ferguson (2010) show that when we change the negative sentence form but not the propositional content, participants no longer show a response advantage for the picture that is consistent with the positive counterpart. Here, $250 \mathrm{~ms}$ after the presentation of a simple negative sentence (e.g. "John hasn't ironed his shirt"), participants responded faster to a picture that is consistent with the positive argument of negation (an ironed shirt), but that $250 \mathrm{~ms}$ after the presentation of a cleft negative sentence (e.g. "It is John who hasn't ironed his shirt"), participants responded faster to a picture that is consistent with the negative argument (a crumpled shirt). Tian et al. argue that the change of linguistic form to a cleft sentence causes a change of accommodated context.

Why is the positive argument often represented during negation processing? The literature offers two perspectives: rejection-based accounts and contextual views. The first perspective draws on the analysis of negation as an external truth-functional operator. Negation reverses the truth value of its embedded proposition. Based on this function, some theories state that a negative sentence is represented by multiple constituents, namely the negation operator and its positive argument. In the course of sentence comprehension or verification, participants first represent the embedded argument, and then reject it or reverse its truth value. Both propositional theories (Clark \& Chase, 1972; Carpenter \& Just, 1975), and the two-step simulation approach (Kaup, Zwaan, et al., 2007) follow the idea of "rejection", although they differ in how the constituents are represented. These theories explain why the positive argument is 
activated in the first place and that this is the cause of the extra difficulty of negation which is often reported in the psycholinguistics literature. They also claim that processing is initially insensitive to negation. By contrast, the second perspective, stemming from Wason (1965), suggests that with the right kind of contextual support, negative sentences are not difficult. In this tradition, Nieuwland and Kuperberg (2008) suggest that, with the right contextual support, the positive argument need not be represented for comprehension. Similar conclusions are drawn in Dale and Duran (2011). Contextual views explain why the positive argument is sometimes not activated when negative sentences have contextual support.

What seems to be missing from the contextualist perspective is an explicit account of the mechanism of context projection that can also explain why the positive argument is sometimes represented when we process negative sentences. This is provided by the dynamic pragmatic account: the positive argument is represented due to QUD accommodation (Tian et al., 2010; Tian \& Breheny, 2015).

Current approaches to natural language interpretation are dynamic - assuming that language use functions to update an information state. In language use, information states contain background information relevant to resolve presuppositions (Stalnaker 1978; Clark 1996) and also information that bears on how the utterance is meant to be relevant (Grice 1989; Sperber \& Wilson 1986). Current dynamic accounts describe the source of relevance of an utterance in terms of a set of salient Questions Under Discussion (QUDs - Ginzburg 2012; Roberts 2012). The linguistic form of a sentence contains cues (e.g. prosodic focus) for the intended QUD (Ginzburg, 2012; Roberts, 2012). When contextual information is implicit or absent, we use these cues to retrieve and accommodate the likely source of relevance, or QUD, addressed by the current sentence (ibid). Tian et al. (2010) argue that negation is a cue for the prominent QUD. 
Without context or further cues, the most prominent QUD for a negative sentence "not $\mathrm{p}$ " is the positive question whether $p$. The prominence of this positive QUD comes from the most frequent use of negation: denial and rejection (Tottie, 1991). When processed out of context, negation often triggers the participants to accommodate a positive QUD. For example, for a simple negative sentence such as "John hasn't ironed his shirt”, its most prominent QUD is whether the positive counterpart is true, namely, whether John has ironed his shirt. We argue that it is QUD accommodation that accounts for why studies often report the representation of content consistent with the positive counterpart when processing a negative sentence, as most studies presented participants with sentences without context. However, if the linguistic form of a negative sentence points to a negative QUD, participants should no longer represent the positive argument. For example, for a cleft negative sentence "It is John who hasn't ironed his shirt", the most prominent QUD is who hasn't ironed their shirt. In this case, comprehenders do not first activate the representation of an ironed shirt. The pattern of results from Tian et al. (2010) who used stimuli such as these is described above and it cannot easily be accounted for using a rejection-based model since in both conditions the same negative proposition is expressed. The results are predicted by our QUD accommodation account.

When does QUD accommodation occur? Given that after reading simple negative sentences participants respond faster to the positive image, results in Tian et al (2010) might suggest that the likely source of relevance (QUD) is inferred before the proposition expressed is represented, akin to a two-step process. However, we argue that dynamic updating processes are fully incremental and interactive. I.e. inferences about both the likely QUD and the likely content are processed incrementally as 
linguistic input proceeds - with inferences about one influencing inferences about the other.

Language processing is incremental and predictive, evidenced by that fact that we can often interact with each other with no gap between conversational turns (Heldner \& Edlund, 2010; Stivers et al., 2009). These properties apply not only to the processing of sentence content, but also to the integration of contextual information. Altmann and Steedman (1988) demonstrates that inferences about how to satisfy the presuppositions of a definite description in a visual context are processed at the same time as - and in turn influences- the computation of inferences about syntactic attachment. Thus we believe that inferences about the likely QUD and the sentence content are processed in parallel. For the items in Tian et al. (2010), we believe the time course of inferring negative content was later than for positive QUDs due to the extra cost of inferring the negative state of affairs from the linguistic stimulus alone. I.e. for, "The shirt is not ironed", the linguistic stimulus itself provides information about the positive state of affairs, while the negative state of affairs (being crumpled) needs to be inferred on the basis of world knowledge that can be activated only after processing the predicate. Our visual world study is set up to eliminate this disadvantage for inferring the negative state of affairs by providing images consistent with both positive and negative states of affairs, available for $1 \mathrm{~s}$ before the onset of the linguistic input. According to the incremental QUD accommodation account, we predict that the timecourse of representing QUDs and content should be comparable in these cases where either is positive or negative.

\section{The Current Study}

The current study investigates when contextual accommodation occurs, and at what point the meaning of negation is incorporated. We compare the time course of 
representations during the processing of positive and negative sentences, using a visual world eye-tracking paradigm. Prior studies in visual world eye-tracking (Cooper, 1974; Tanenhaus, Spivey-Knowlton, Eberhard, \& Sedivy, 1995) found that even without any task other than reading for comprehension, participants shift their visual attention around the scene as the linguistic stimuli unfold. Altmann and colleagues (Altmann \& Kamide, 1999; Altmann \& Kamide, 2007) found that language-mediated eye movements are anticipatory, and they correspond to a dynamically changing representation of events. Altmann et al. (2007) presented participants with semi-realistic visual scenes such as a man standing next to table with an empty wine glass, a full beer glass and some distractors, while listening to a sentence such as "the man will drink all of the beer" or "the man has drunk all of the wine". In this "look and listen" task, they found that participants shifted their visual attention to the full beer glass or empty wine glass before the onset of the critical noun "beer" or "wine". This shows that participants incrementally update their representation of events by combining linguistic and visual information.

The look-and-listen paradigm provides us with a tool to study the time course of the processing of negative sentences compared to their positive counterparts. As in Tian et al. (2010) participants hear positive and negative versions of a sentence in simple, (1), and clefted (2) formats:

1a) John has ironed his brother's shirt. (simple positive)

1b) John hasn't ironed his brother's shirt. (simple negative)

2a) It is John who has ironed his brother's shirt. (cleft positive)

2b) It is John who hasn't ironed his brother's shirt. (cleft negative)

Positive sentences like 1a) and 2a) imply that the current state of the shirt is smooth, while their negative versions $1 b$ ) and $2 b$ ) imply that the shirt is crumpled. 
Following Altmann and Kamide (2007), in our study, participants hear sentences while looking at a visual scene containing the representation of the implied state (the target) and the representation of the opposite state (the competitor). According to rejection accounts, both simple and cleft negatives ( $1 \mathrm{~b}$ and $2 \mathrm{~b}$ ) should be processed by first representing the positive argument (John has ironed his brother's shirt), thus predicting a delay in $1 b$ ) relative to $1 a)$, and similarly in $2 b$ ) relative to $2 a)$. For both negative sentences, attention should first be directed to the representation of the positive argument (competitor), before being shifted to the target.

By contrast, the incremental QUD accommodation account claims that participants will incrementally update predictions about content and QUD in parallel. As established in Tian et al., (2010), without further context, both simple positive and negative sentences of the form, "NP1 has/hasn't V NP2" are liable to be associated with a QUD about the positive state of affairs, "whether NP1 has V-ed NP2”. For the items in 1a) and 1b), given a visual context showing a shirt in a crumpled state and a shirt in a smooth state, participants ought to start predicting both sentence content and sentence QUD from the offset of the verb. For subject-clefted sentences ("It was NP who has/hasn't V NP"), our assumption is that the most likely QUD is of the form, who/what has/hasn't VNP, based on the presupposition of the sentence, someone/something has/hasn't VNP. Therefore, in incremental processing, by the offset of the auxiliary "has"/ "hasn't", participants should have established the syntactic form as a subjectclefted sentence, and thus be able to anticipate the general form of both the presupposition and the QUD. By the verb "ironed", given the visual context, participants ought to be able to anticipate both the QUD for the sentence and its content. Thus, for all of the items in the simple and cleft conditions of this experiment, participants are expected to be able to predict both content and QUD from the same 
point in the linguistic stimulus. Thus we predict that in the simple negative, (1b), participants will look at both the negative target and its positive competitor before focusing on the content of the assertion. In contrast, since content and QUD are both positive for the 1a), we predict a rapid bias to the positive target. For the clefted version of the propositions ( $2 \mathrm{a}$ and $2 \mathrm{~b}$ ), we predict little or no delay of the negative with respect to the positive. This is so since for both positive and negative cleft sentences, the likely QUD is of the same polarity as the content. For the positive (2a), the prominent QUD is who ironed their brother's shirt, while for the negative (2b) it is who did not iron their brother's shirt.

While clefted items are predicted to give rise to a target bias in the same time course as each other, we do not predict that bias to target will form in the same time course as in the simple positive. This is so since the clefted form is less frequent than the simple form and it is linguistically and pragmatically more complex. In particular, the presupposition of the cleft construction tends to suggest a more complex situation than is suggested for the simple assertions: one which involves people other than John and his brother and also multiple shirts. Other things equal, such situational background would take more resources to construct (cf Altmann \& Steedman, 1988). In addition the presupposition or QUD of the sentence would typically give rise to a conversational implicature $^{2}$ that the predicate does not hold for others in the context. That is, for (2a) it may be inferred as background information that others did not iron their brother's shirt and for $(2 b)$ that someone did iron their brother's shirt. To the extent that these implicatures are accessed in the same time course as sentence content (Grodner et al., 2010; Breheny, Ferguson, \& Katsos, 2013) we should see a diminution of the rate of bias formation of both types of cleft sentence relative to the simple sentence ${ }^{3}$. 


\section{Methods}

\section{Participants}

Thirty-six participants between the age of 19 and 36 were recruited from University College London via an online psychological subject pool, 20 were female. They participated either for course credit or £4. All participants speak English as a native language. They have uncorrected or corrected to normal vision.

\section{Materials}

This experiment has a two by two within participants design. The two independent variables are polarity and cleft-ness. These two variables generate four experimental conditions: simple positive, simple negative, cleft positive and cleft negative. 40 experimental items were constructed (see Table 1 for examples). All predicates of experimental sentences described bi-polar states of the same object, such as "iron the shirt" (the shirt is either ironed or not) and "turn on the TV" (the TV is either on or off). Thus, positive and negative versions of the predicate each imply a unique state, which is supported by the available visual context (depicting these two alternate states). Experimental sentences are of the form of “(It is) Name (who) has/hasn't verb his/her someone's noun". For example, "Matt hasn't shut his dad's window" (simple) or "It is Matt who hasn't shut his dad's window" (cleft). Note that we added words such as "his dad's" ${ }^{\prime 4}$ in between the verb and target noun, because previous studies (Altmann \& Kamide, 2007 experiment 1; Barr, 2008a) have demonstrated the need to allow participants more time to anticipate referents before bottom-up information is available.

Each experimental item generates four sentences, and four lists are created, each containing 40 experimental sentences. Each item only appears once in each list, in one of the four conditions, using a Latin-square design. In addition, there are 40 fillers, 
among which half were positive, and half were negative. Half indicate the beginning state of an event (will and should have), while the other half indicate the end state of an event (shouldn't have). After 20 sentences (10 experimental), there was a

comprehension question. The aim was to check whether the participants understood the content of the sentence. For example, for the filler "Tom has fixed his uncle's fridge", the question was “is Tom's uncle's fridge still broken?”. Each participant heard 80 sentences in total (see Table 1 for examples).

(Table 1 about here).

Sentences were recorded by a male speaker of Southeast British English. The speaker was instructed to read all sentences with a natural intonation, while putting a stress on "has" or "hasn't" for simple sentences, and on the name (e.g. John) for cleft sentences. Note that in cleft sentences, "hasn't" received a secondary stress, but "has" did not. This was not instructed.

Each experimental item and each filler sentence is paired with a visual scene consisting of five items: a person (which matches the gender of the name), two critical images and two distractors. The two critical images include a target and a competitor. The target represents the implied state of the item, while the competitor represents the opposite state. For example, for the sentence "Matt hasn't shut his dad's window", the target is an open window and the competitor is a shut window. The target for a negative sentence is the competitor for the positive counterpart. The two distractors are images of a different item in two states (for example a plain bagel and a bagel with cream cheese), so that participants will not be able to predict the verb before hearing it. All pictures of the person measure $150 * 250$ pixels. All pictures of four items measure $250 * 250$ pixels. The screen resolution is $1024 * 768$ pixels. The picture of the person is always in the 
centre of the screen. Target, competitor and two distractors are located in the four corners of the screen but the exact location of each is counterbalanced.

\section{Procedure}

The experiment was conducted using E-Prime software and a Tobii X60 eye-tracker. Participants were calibrated at the beginning of the experiment using a nine-point display. Head movements were not restricted but participants were asked to stay still as much as possible throughout the duration of the experiment. Before each trial, there was a fixation cross in the centre of the screen, and participants' eye gaze had to be fixed on this point for a continuous 3 seconds before the trial started. Then a scene with five images (as described above) appeared on the screen. Participants had one second to preview the images, and the audio stimuli started after the preview. During the audio, the participants were instructed to simply listen and look at the images. The sentences last an average of 3.04 seconds (standard deviation 0.37 seconds, minimum length 2.31 seconds, maximum length 4.35 seconds). Eye movements were recorded for 6 seconds for each trial. For 20 out of 80 sentences, a comprehension question appeared on the screen after the sentence, and participants pressed either the "yes" or "no" key to answer the question (they are 1 and 0 , with stickers which says "yes" or "no"). The whole experiment lasted approximately 25 minutes.

\section{Data Analysis and Results}

\section{Analysis of audio stimuli}

The onset and offset for each word in the experimental audios are hand marked using phonetics analysis software Praat (Boersma \& Weenink, 2013) with a millisecond resolution. For the analysis, we are interested in the main verb, post-verb silence ${ }^{5}$, 
possessive pronouns ("his" or "her"), second possessive, such as "brother's" or "friend's", and the final noun. Table 2 shows the mean and standard deviation of duration for each of these words in milliseconds.

(Table 2 about here).

Between positive and negative simple sentences, there is no significant difference in the duration of the verb $(\mathrm{t}=0.34, p=0.74)$, "his"/"her" $(\mathrm{t}=0.16, p=$ $0.87)$, "someone's" $(\mathrm{t}=1.96, p=0.06)$, or the noun $(\mathrm{t}=1.83, p=0.07)$. There is a significant difference in the duration of the post-verb silence $(\mathrm{t}=2.98, p=0.02)$. Between positive and negative cleft sentences, there is no significant difference in the duration of "his"/"her" ( $\mathrm{t}=0.17, p=0.87)$, or "someone's" $(\mathrm{t}=0.22, p=0.83)$. There is a small but significant difference in the duration of the verb $(\mathrm{t}=2.65, p=0.01$; negative $>$ positive), in post-verb silence $(\mathrm{t}=0.25, p=0.02$; negative $>$ positive $)$, and in noun $(\mathrm{t}$ $=0.25, p=0.01$; negative $>$ positive). Overall the durations of verb-to-noun window should be comparable between simple positives and negatives, and between cleft positives and negatives (overall differences at $1.4 \%$ of the verb to noun window for simple conditions, and at $5 \%$ for cleft conditions). Therefore to compare simple positives with simple negatives, and to compare cleft positives with cleft negatives, we decided to analyse both averages within word regions, and averages during every $100 \mathrm{~ms}$ time slices in a $1000 \mathrm{~ms}$ time window.

Comparing simple and cleft sentences, there is no significant difference in the duration of "his"/"her" ( $\mathrm{t}=1.35, p=0.18)$, "someone's" $(\mathrm{t}=1.94, p=0.06)$, or the noun region $(\mathrm{t}=0.21, p=0.84)$. There is a significant difference in the duration of verb $(\mathrm{t}=-$ $6.23, p<0.001)$. On average the verbs in simple sentences are $49 \mathrm{~ms}$ longer than cleft. Post-verb silence in simple sentence is $158 \mathrm{~ms}$ longer than in cleft $(\mathrm{t}=11.78, p<$ 0.001). The duration differences ( $14 \%$ of the verb to noun window) make comparing 
averages in word regions between simple and cleft less optimal. Therefore we did a fixed window analysis to compare simple with cleft sentences.

\section{Analysis of eye-movements: main analysis}

Fixations that landed within the coordinates of the target and competitor are analysed against key time periods in the audio stimuli. Fixations that landed within the coordinates of two distractors and the image of the person are also extracted. Any fixations deemed invalid due to blinking or head movements were removed. Any fixations shorter than 80 milliseconds were excluded, as extremely short fixations are often due to false saccade planning (Rayner \& Pollatsek, 1989). Table 3 shows the percentages of fixations to each area of interest against all fixations across different word regions.

We are interested in the anticipatory looks to the target compared to the competitor during the period following the verb. Thus, we calculated the probability of looks to the target and competitor as a function of time, using the log-ratio measure: $\mathrm{Ln}\left(\mathrm{P}_{\text {target }} / \mathrm{P}_{\text {competitor }}\right)$. $\mathrm{P}_{\text {target }}$ refers to the proportion of looks to target image, and $\mathrm{P}_{\text {competitor }}$ refers to the proportion of looks to the competitor image ${ }^{6}$ " $L n$ " is the natural $\operatorname{logarithm}{ }^{7}$. The measure is symmetrical around zero such that a bias towards the target is reflected in a positive log-ratio score and a bias towards the competitor is reflected in a negative log-ratio score. A log-ratio of 0 shows that there is an equal probability of looks to the target and competitor objects. This single 'target advantage' DV therefore provides a direct comparison between looks to the target versus competitor and was chosen based on related research that has used similar methods (Arnold, Eisenband, Brown-Schmidt, \& Trueswell, 2000; Brown-Schmidt, Gunlogson, \& Tanenhaus, 2008; Ferguson, Scheepers, \& Sanford, 2010; Ferguson \& Breheny, 2011, 2012; Heller, Grodner, \& Tannenhaus, 2008). 
Figure 1 (simple) and Figure 2 (cleft) plot the target advantage score over each $17 \mathrm{~ms}$ sample (the eye tracker runs at $60 \mathrm{~Hz}$ ), from the onset of the verb to the end of the sentence. With regard to the example sentence “(It is) Matt (who) hasn't shut his dad's window", the figures cover before, during and after the section "shut his dad's window". The regions are named as verb, [pause], his, someone's, and noun. In these graphs, vertical lines represent the average onsets and offsets of key regions. However, for calculations of percentage of looks and statistical tests, word regions for each sentence is defined by the onsets and offsets of words for that particular sentence. Note that for all plots and data analysis, word regions have been offset by $200 \mathrm{~ms}$, as it takes around 200ms to launch an eye-movement (Hallett, 1986). As sentences differ in their onsets and offsets of words, the curves in Figure 1 and Figure 2 are resynchronized at the onset of each word, so that the graph more accurately reflects the evolving visual biases relative to the audio stimuli (Altmann \& Kamide, 2009). The 500ms pre-verb window is synced to the onset of the verb.

(Figure 1 about here).

(Figure 2 about here).

We averaged the target advantage scores in key regions both by participant and by item. Statistical tests are applied on these average target advantage scores. Note that

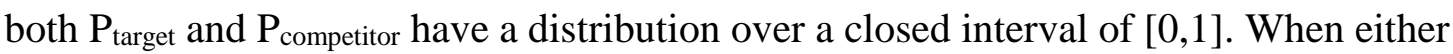
measurement is 0 , it is a problem for $\log$ transformation. In this case, we transformed 0 values using the function $\mathrm{y}^{\prime}=[\mathrm{y}(\mathrm{N}-1)+\mathrm{s}] / \mathrm{N}$ (Smithson \& Verkuilen, 2006, see supplementary material). $\mathrm{Y}^{\prime}$ is the transformed value, $\mathrm{y}$ is the original value. $\mathrm{N}$ is the sample size and $\mathrm{s}$ is a constant between 0 and 1 . From a Bayesian point of view, $\mathrm{s}$ acts as if we are taking a prior into account. 0.5 is recommended as a reasonable choice for s. This function "squeezes" the values into an open interval $(0,1)$. 
Let us inspect the results in the Figures. Here we focus on the target advantage scores between the offset of the verb and the offset of the noun. During and before the verb region, no difference between looks to the target and the competitor was expected or observed (see percentages of looks in Table 3 and t-test statistics in Table 4). The low proportion of looks to the target and the competitor in the early regions $(<15 \%)$ meant that small fluctuations in the percentages of looks could lead to large fluctuations in target advantage scores. Note that initial analyses examine the time course of effects separately for simple and cleft sentences due to differences in the length of word-based time-regions of analysis (i.e. the verb and post-verb silence are approximately $200 \mathrm{~ms}$ longer in the simple versus cleft sentences).

For simple sentences (Figure 1) there is a difference between positive and negative conditions from the offset of the verb onwards. For positives, a bias towards the target was formed immediately after the verb. For negatives, however, there are roughly equal amount of looks to the target and the competitor after the verb, in the post-verb silence and "his" regions. A target bias did develop later, crucially before the onset of the noun (during "someone's"). A 3 (region: post-verb silence and "his" vs. "someone's" vs. noun) by 2 (polarity: positive $v s$. negative) ANOVA shows that there is no significant region by polarity interaction $\mathrm{F}_{1}(2,70)=1.17, p=0.32, \eta_{\mathrm{p} 2}=0.03$; $F_{2}(2,76)=0.28, p=0.76, \eta_{\mathrm{p} 2}=0.007$. However, there is a significant main effect of region: $\mathrm{F}_{1}(2,70)=3.54, p=0.04, \eta_{\mathrm{p} 2}=0.03 ; \mathrm{F}_{2}(2,76)=5.74, p=0.005, \eta_{\mathrm{p} 2}=0.13$ (reflecting increasing looks to the target over time), and importantly, a highly significant main effect of polarity: $\mathrm{F}_{1}(1,35)=32.03, p<0.001, \eta_{\mathrm{p} 2}=0.49 ; \mathrm{F}_{2}(1,38)=$ $13.57, p=0.001, \eta_{\mathrm{p} 2}=0.26$. This suggests that participants were consistently more likely to fixate the target (versus the competitor) following a positive sentence compared to a negative sentence from the verb offset to the noun offset. We then 
performed planned paired-sampled t-tests to compare positive and negative conditions using the target advantage scores in the verb and post-verb regions (see Table 4).

Results show that the difference between positive and negative conditions is significant in the post-verb silence and "his", "someone's", and the noun region.

In the case of cleft sentences (Figure 2), there is no difference between positive and negative conditions from the offset of the verb to the onset of the noun. In this period, participants were paying comparable attention to the target and the competitor. A 3 (region: post-verb silence and "his" vs. "someone's" vs. noun) by 2 (polarity: positive vs. negative) ANOVA shows that there is no significant region by polarity interaction $\mathrm{F}_{1}(2,70)=1.17, p=0.32, \eta_{\mathrm{p} 2}=0.03 ; \mathrm{F}_{2}(2,76)=0.74, p=0.48, \eta_{\mathrm{p} 2}=0.02$, however, there is a significant main effect of region: $\mathrm{F}_{1}(2,70)=3.54, p=0.04, \eta_{\mathrm{p} 2}=$ $0.03 ; \mathrm{F}_{2}(2,76)=12.0, p<0.001, \eta_{\mathrm{p} 2}=0.24$ (again showing increasing looks to the target over time). Crucially, unlike the simple condition, here polarity does not have a significant main effect (though trending by participants): $\mathrm{F}_{1}(1,35)=3.89, p<0.06, \eta_{\mathrm{p} 2}=$ $0.10 ; \mathrm{F}_{2}(1,38)=1.04, p=0.31, \eta_{\mathrm{p} 2}=0.027$. Planned paired sampled t-tests on target advantage scores (see Table 4) show that there is no difference between positive and negative conditions during the post-verb silence and "his", or "someone's". In the noun region, the difference is significant by subjects only.

Thus, comparing simple and cleft sentences, between the offset of the verb and the onset of the noun, there is a difference between positive and negative conditions for simple but not cleft sentences. In order to test whether this interactive pattern between sentence types is significant, we need to extract a fixed length window from the offset of the verb for both simple and cleft sentences. This is because the post-verb silence region for cleft is shorter than that for simple by $160 \mathrm{~ms}$, thus regions defined by word boundaries are not ideal for comparison between simple and cleft. We therefore 
extracted a $449 \mathrm{~ms}$ window from the offset of verb for all items. This is the shortest gap between the offset of the verb and the onset of the noun among all items. As before, we calculated the average target advantage score (i.e. proportion of looks to the target over competitor) in the post-verb $449 \mathrm{~ms}$ window. We performed a 2 (cleftness: simple $v s$. cleft) by 2 (polarity: positive $v s$. negative) ANOVA, which shows that there is a significant cleftness by polarity interaction over this time period, $\mathrm{F}_{1}(1,35)=8.19, p=$ $0.007, \eta_{\mathrm{p} 2}=0.19 ; \mathrm{F}_{2}(1,38)=6.16, p=0.018, \eta_{\mathrm{p} 2}=0.14$. Paired sample t-tests show that there is a highly significant difference between simple positive and negative: $t_{1}(35)=$ 4.53, $p=6.6 \mathrm{e}-05, \mathrm{t}_{2}(35)=3.02, p=0.004$; but no difference between cleft positive and negative: $\mathrm{t}_{1}(35)=0.24, p=0.81, \mathrm{t}_{2}(35)=0.49, p=0.63$.

Finally, in order to determine whether there are significantly more looks to the target than the competitor, i.e. whether the average target advantage score is significantly greater than zero, we performed planned one-sampled t-tests comparing target advantage scores to zero for each of the four word-based regions (verb, post-verb silence and "his", "someone's", and noun; Table 5). Results show that for simple sentences, positive conditions elicit a significant bias to the target immediately after the verb, while in negative conditions, the bias to the target only becomes significant in the "noun" region. For cleft sentences, positive and negative conditions show similar patterns: there is a bias to the target during "someone's" region (significant by subject only for cleft negative) and the noun region.

(Table 3 about here).

(Table 4 about here).

(Table 5 about here).

\section{Time-course analysis}

In order to determine exactly when a target bias was established, we conducted a time- 
course analysis on a one-second time period from the offset of the verb. We divided this period into ten $100 \mathrm{~ms}$ time slices, and calculated a target advantage score for each time point and condition. Figure 3 and Figure 4 plot the averaged target advantage scores for 10 time slices from the offset of verb, for simple sentences and cleft sentences respectively. They show that shortly after the verb (in the first 5 slices), target advantage scores differ greatly between simple positives and negative, but are almost identical between cleft positive and negative. Later on (from slice 6 onwards), target advantage scores for positive and negative sentences differ in both the simple condition and the cleft condition. We performed a 10 (time bin) by 2 (cleftness: simple vs. cleft) by 2 (polarity: positive $v s$. negative) ANOVA, which showed a time by clefting by polarity interaction (significant by participants): $\mathrm{F}_{1}(9,315)=2.8, p=0.04, \eta_{\mathrm{p} 2}=0.074$; $\mathrm{F}_{2}(9,342)=1.54, p=0.20, \eta_{\mathrm{p} 2}=0.04$. To investigate this further we conducted separate 10 (time bin) by 2 (polarity: positive $v s$. negative) ANOVAs for simple sentences and cleft sentences. Here, simple sentences showed a significant time by polarity interaction: $\mathrm{F}_{1}(9,315)=2.42, p=0.036, \eta_{\mathrm{p} 2}=0.45 ; \mathrm{F}_{2}(9,342)=2.47, p=0.03, \eta_{\mathrm{p} 2}=$ 0.43 , however the time by polarity interaction was not significant for cleft sentences (but showed a trend by participants): $\mathrm{F}_{1}(9,315)=2.22, p=0.053, \eta_{\mathrm{p} 2}=0.43 ; \mathrm{F}_{2}(9,342)$ $=1.09, p=0.4, \eta_{\mathrm{p} 2}=0.25$.

To determine the point at which a reliable target bias was formed, we performed one-sampled t-tests comparing target advantage scores with zero for each time slice (Table 6 , reporting both the original $\mathrm{p}$ values and the Šidák corrected $p$ values ). Results show that for simple positive sentences, the target bias was significant from $200 \mathrm{~ms}$ after the offset of the verb. For simple negatives, the target bias has trending significance only in the 10th time slice (see Figure 3). For cleft sentences, the target bias becomes significant in the 6th time slice for cleft positives (trending in the 5th). For cleft 
negatives, the target bias is significant by subject in the 6th and 7th slices and significant by item in the 9th slice (see Figure 4). The results further demonstrate the difference in processing time between simple positive and negative, and the lack of difference in processing time between cleft positive and negative.

(Table 6 about here).

(Figure 3 about here).

(Figure 4 about here).

\section{General Discussion}

This study shows that shortly after the verb in simple negative sentences like "Matt hasn't shut his dad's window", participants paid comparable attention to both the image consistent with the content of the positive counterpart (a shut window) and the image consistent with the negative sentence meaning (an open window). This suggests that when processing simple negative sentences, the content of the positive counterpart is initially activated. One might argue that the initial lack of difference between looks to the target and the competitor in the simple negative condition was due to participants looking randomly at the two pictures, i.e. they never activated the positive argument. However, as many previous studies have found that the positive argument is often initially activated during negation processing, our interpretation is more plausible. However, within 900ms from the offset of the verb (in the noun region), participants had shifted their attention away from the positive content, and focused on the negationconsistent representation. In comparison, when hearing a simple positive sentence like "Matt has shut his dad's window", participants favoured the target representation (shut window) immediately from verb offset.

In the case of cleft sentences, bias to an image consistent with the sentence content forms at the same rate for negative and positive cases. When hearing either a 
positive or negative cleft sentence, participants pay comparable attention to both the target and the competitor representation after the verb. For cleft positives, a target bias became significant at around 500ms after the offset of the verb, and for cleft negatives, it took around 600ms. Participants' attention shifted away from the competitor and onto the target in "dad's" region (as in "his dad's window"), before the onset of noun. The time-course of the processing of cleft negatives is very similar to cleft positives. As predicted, Cleft sentences of both polarities experienced some delay compared to the simple positive sentence, due to the greater complexity of the situational context and the possible presence of a conversational implicature arising from the cleft construction.

Comparing results from simple and cleft sentences, there is a difference between simple but not cleft sentences, as demonstrated by the significant polarity-by-cleftness interaction in the $449 \mathrm{~ms}$ post-verb window. What's more, despite the fact that cleft negatives are linguistically and pragmatically more complex than simple negatives, the formation of the target bias did not take longer in cleft negatives than simple negatives. These results suggest that the processing delay in simple negatives is not in fact caused by the first step of negation processing. Rather, it is likely due to QUD accommodation. Without context or further cues, the most prominent QUD for a simple negative sentence is whether the positive counterpart is true, e.g. whether Matt has shut his dad's window. Accommodating this QUD results in the representation of content consistent with the positive counterpart. In contrast, a cleft negative sentence has a negative prominent QUD, such as who hasn't shut their dad's window. The representation of this QUD is congruent with the representation of the sentence meaning. Similarly, a cleft positive sentence has a positive prominent QUD, such as who has shut their dad's window, which is also congruent with the representation of the sentence meaning. 
Therefore, the timecourse of bias formation for a negative cleft sentence is no more delayed than that for a positive cleft sentence.

\section{Evaluation of current results against rejection accounts}

Our results are incompatible with rejection accounts in at least three predictions. First, according to rejection accounts, we should see that for both simple negative and cleft negative sentences the formation of a bias to the target is delayed compared to their positive counterparts, given that both types of negative sentence express the same proposition. Instead, we saw that only simple negatives are delayed compared to their positive counterparts. Second, rejection accounts should predict that when processing a negative sentence participants FIRST represent content consistent with the positive counterpart and then represent the state of affairs consistent with sentence meaning. Instead, we saw that when hearing a simple negative sentence, participants paid comparable attention to both the representation for the positive counterpart and the negation consistent representation, before shifting attention away from the positivecounterpart representation. This suggests that representation of the positive-counterpart content is not a discrete first step, but happens in parallel with representing the sentence meaning. Third, rejection accounts predict that the meaning of negation is incorporated after the positive argument is processed ${ }^{8}$. However, our results for cleft negatives suggest that the meaning of negation can be incorporated incrementally. A target bias was formed just $600 \mathrm{~ms}$ after the offset of the verb, before the onset of noun. The timecourse is similar to cleft positives. This suggests that participants start combining the meaning of negation with the verb as soon as they hear the verb, namely "not shut" implies "open". This information is then used to infer the shape/state of the target object, and direct their visual attention to the object that is compatible with the combined meaning of the negation, the verb and the noun. 


\section{The representation of the positive content}

Coming back to the literature on the activation of the content of the positive counterpart during negative sentence processing, our results for simple negative sentences support previous findings that the positive-counterpart content can be activated in the early stage of negative sentence processing. The early occurrence of this representation supports the idea that QUD accommodation happens incrementally during sentence processing.

In terms of the duration of the activation of the positive content, in our visual world paradigm, it lasted for around $800 \mathrm{~ms}-900 \mathrm{~ms}$. This duration is compatible with the findings of Hasson and Glucksberg (2006) as well as Kaup, Lüdtke and Zwaan (2006).

Our results also show that the activation of the positive-counterpart content is not a discrete first step, but happens in parallel with the activation of sentence-meaning consistent representation. The results from most other studies have no support for this. They generally found higher accessibility of the positive content than the negationconsistent representation during the early stages. How can we reconcile our results with these findings? In our paradigm all potential representations are visually present on the screen. Without such visual stimuli, it is highly likely that representing the state of affairs consistent with the negative sentence takes longer than representing the positive content, as the former involves an extra inferential step. For example, to form a representation based on the stimulus, "the window is not open", participants must infer that the window is closed and represent such a state, whereas to form a representation for, "the window is open", no additional inference is required beyond access to the meanings of the predicates. Thus, in paradigms where representations are not shown in 
advance, participants may first arrive at the representation of the QUD since the positive content is easier to access.

Overall our data in simple sentences support previous findings that the positivecounterpart content can be activated during negation processing.

\section{Implications}

The current study presents the first exploration of negation processing using a visual world paradigm that allows us to track the time-course of inferences based on positive and negative framed statements. Moreover, it provides an extension of previous work (Tian et al., 2010) to establish how sentence structure, specifically manipulating sentence focus using clefts, influences language processing and facilitates representation of the negated argument. Beyond the processing of negation, this study relates to the broader question of how pragmatic information is incrementally updated during sentence processing. Research has grown in the online integration of pragmatic information. For example, we can integrate common ground and the speaker's epistemic state at the earliest moment and use such information to predict upcoming referents (Breheny et al., 2013; Heller et al., 2008); we can access scalar implicatures on-line with little or no delay (Breheny, Ferguson, \& Katsos, 2012; Grodner, Klein, Carbary, \& Tanenhaus, 2010); we can infer information about the speaker using accents and cultural heuristics, and use it to anticipate upcoming words in a sentence (van Berkum, van den Brink, Tesink, Kos, \& Hagoort, 2008). However, as far as we know, there have been no prior studies in the online accommodation of QUD. We have shown that the linguistic form of a sentence contains cues for how it is related to the prior context. On hearing or reading a sentence when there is insufficient or no context, comprehenders do not just process the semantic meaning of a sentence, rather, they also use cues to infer and accommodate a likely context, specifically a QUD. This process is automatic 
and incremental. Negation is one such cue for retrieving a prominent QUD. Without other cues (such as cleft construction), the most prominent QUD for a negative sentence is positive. This is why studies often report the representation of the positivecounterpart content in negation processing. 


\section{References}

Altmann, G., \& Kamide, Y. (1999). Incremental interpretation at verbs: restricting the domain of subsequent reference. Cognition, 73(3), 247-64. doi:10.1016/s00100277(99)00059-1

Altmann, G., \& Kamide, Y. (2007). The real-time mediation of visual attention by language and world knowledge: Linking anticipatory (and other) eye movements to linguistic processing. Journal of Memory and Language, 57(4), 502-518. doi:10.1016/j.jml.2006.12.004

Altmann, G., \& Kamide, Y. (2009). Discourse-mediation of the mapping between language and the visual world: eye movements and mental representation. Cognition, 111(1), 55-71. doi:10.1016/j.cognition.2008.12.005

Altmann, G., \& Steedman, M. (1988). Interaction with context during human sentence processing. Cognition, 30(3), 191-238.Arroyo, F. (1982). Negatives in context. Journal of Verbal Learning and Verbal Behavior, 21(1), 118-126. doi:10.1016/0010-0277(88)90020-0

Arnold, J.E., Eisenband, J., Brown-Schmidt, S. \& Trueswell, J.C. (2000). The rapid use of gender information: Evidence of the time course of pronoun resolution from eyetracking. Cognition, 76, B13-B26. doi:10.1016/s0010-0277(00)00073-1

Barr, D. J. (2008a). Pragmatic expectations and linguistic evidence: Listeners anticipate but do not integrate common ground. Cognition, 109(1), 18-40. doi:10.1016/j.cognition.2008.07.005

Barr, D. J. (2008b). Analyzing "visual world" eyetracking data using multilevel logistic regression. Journal of Memory and Language, 59(4), 457-474. doi:10.1016/j.jml.2007.09.002 
Boersma, P., \& Weenink, D. (2013). Praat: Doing Phonetics by Computer (Version 5.3. 49). doi:10.1097/aud.0b013e31821473f7

Breheny, R., Ferguson, H. J., \& Katsos, N. (2012). Investigating the timecourse of accessing conversational implicatures during incremental sentence interpretation. Language and Cognitive Processes, 28(4), 443-467. doi:10.1080/01690965.2011.649040

Breheny, R., Ferguson, H. J., \& Katsos, N. (2013). Taking the epistemic step: toward a model of on-line access to conversational implicatures. Cognition, 126(3), 423-40. doi:10.1016/j.cognition.2012.11.012

Brown-Schmidt, S., Gunlogson, C., \& Tanenhaus, M. K. (2008). Addressees distinguish shared from private information when interpreting questions during interactive conversation. Cognition, 107(3), 1122-1134. doi:10.1016/j.cognition.2007.11.005

Carpenter, P. A., \& Just, M. A. (1975). Sentence comprehension: A psycholinguistic processing model of verification. Psychological Review, 82(1), 45-73. doi:10.1037/h0076248

Clark, H. (1996). Using Language. Cambridge: Cambridge University Press. doi:10.1017/s0022226798217361

Clark, H. H., \& Chase, W. G. (1972). On the process of comparing sentences against pictures. Cognitive Psychology, 3(3), 472-517. doi:10.1016/0010-0285(72)900199

Cooper, R. (1974). The control of eye fixation by the meaning of spoken language: A new methodology for the real-time investigation of speech perception, memory, and language processing. Cognitive Psychology, 6, 84-107. doi:10.1016/00100285(74)90005-X 
Dale, R., \& Duran, N. D. (2011). The Cognitive Dynamics of Negated Sentence

Verification. Cognitive Science, 35(5), 983-996. doi:10.1111/j.1551-6709.2010.01164.x

Ferguson, H.J. \& Breheny, R. (2012). Listeners' eyes reveal spontaneous sensitivity to others' perspectives. Journal of Experimental Social Psychology, 48, 257-263. doi: http://dx.doi.org/10.1016/j.jesp.2011.08.007

Ferguson, H. J., \& Breheny, R. (2011). Eye movements reveal the time-course of anticipating behaviour based on complex, conflicting desires. Cognition, 119(2), 179-196. doi:10.1016/j.cognition.2011.01.005

Ferguson, H. J., Scheepers, C., \& Sanford, A. J. (2010). Expectations in counterfactual and theory of mind reasoning. Language \& Cognitive Processes, 25(3), 297-346. doi:10.1080/01690960903041174

Fischler, I., Bloom, P. A., Childers, D. G., Roucos, S. E., \& Perry, N. W. (1983). Brain potentials related to stages of sentence verification. Psychophysiology, 20(4), 400409. doi:10.1111/j.1469-8986.1983.tb00920.x

Gelman, A., Hill, J., \& Yajima, M. (2012). Why we (usually) don’t have to worry about multiple comparisons. Journal of Research on Educational Effectiveness, 5(2), 189-211. doi: 10.1080/19345747.2011.618213

Geurts, B. (2010). Quantity Implicature. Cambridge University Press. doi:10.1017/cbo9780511975158

Ginzburg, J. (2012). The Interactive Stance. CSLI: Center. Oxford University Press. doi: 10.1093/acprof:oso/9780199697922.001.0001

Giora, R. (2006). Anything negatives can do affirmatives can do just as well, except for some metaphors. Journal of Pragmatics, 38(7), 981-1014. doi: 10.1016/j.pragma.2005.12.006 
Giora, R., \& Balaban, N. (2005). Negation as positivity in disguise. In H. L. Colston \& A. Katz (Eds.), Figurative language comprehension: Social and cultural influences (pp. 233-258). Hillsdale, NJ: Erlbaum. doi: 10.1017/s0047404506290341

Giora, R., Fein, O., Aschkenazi, K., \& Alkabets-zlozover, I. (2007). Negation in Context: A Functional Approach to Suppression. Discourse Processes, 43(2), 153 172. doi: 10.1207/s15326950dp4302_3

Grice, H.P. (1989). Logic and conversation. In Studies in the Way of Words (pp. 22-40). Cambridge, MA: Harvard University Press. doi: 10.2307/2219730

Grodner, D., Klein, N. M., Carbary, K. M., \& Tanenhaus, M. K. (2010). “Some,” and possibly all, scalar inferences are not delayed: Evidence for immediate pragmatic enrichment. Cognition, 116(1), 42-55. Hallett, P. E. (1986). Eye movements. In K. B. Boff, L. Kaufman, \& J. P. Thomas (Eds.), Handbook of perception and human performance I: Sensory processes and perception (pp. 10-102). New York: Wiley. doi: 10.1016/j.cognition.2010.03.014

Hasson, U., \& Glucksberg, S. (2006). Does understanding negation entail affirmation? An examination of negated metaphors. Journal of Pragmatics, 38(7), 1015-1032. doi: 10.1016/j.pragma.2005.12.005

Heldner, M., \& Edlund, J. (2010). Pauses, gaps and overlaps in conversations. Journal of Phonetics, 38(4), 555-568. doi: 10.1016/j.wocn.2010.08.002

Heller, D., Grodner, D., \& Tanenhaus, M. K. (2008). The role of perspective in identifying domains of reference. Cognition, 108(3), 831-836. doi: 10.1016/j.cognition.2008.04.008

Kaup, B., Yaxley, R. H., Madden, C. J., Zwaan, R., \& Lüdtke, J. (2007). Experiential Simulations of Negated Text Information. The Quarterly Journal of Experimental Psychology, 60, 976-990. doi: 10.1080/17470210600823512 
Kaup, B., \& Zwaan, R. (2003). Effects of Negation and Situational Presence on the Accessibility of Text Information. Journal of Experimental Psychology: Learning, Memory, and Cognition, 29, 439-446. doi: 10.1037/0278-7393.29.3.439

Kaup, B., Zwaan, R., \& Lüdtke, J. (2006). Procesing negated sentences with contradictory predicates: Is a door that is not open mentally closed? Journal of Pragmatics, 38, 1033-1050. doi: 10.1016/j.pragma.2005.09.012

Kaup, B., Zwaan, R., \& Lüdtke, J. (2007). The experiential view of language comprehension: How is negated text information represented? In F. Schmalhofer \& C. A. Perfetti (Eds.), Higher level language processes in the brain: Inference and comprehension processes (pp. 255-288). Mahwah, NJ: Lawrence Erlbaum. doi: $10.4324 / 9780203936443$

Levine, M. (1966). Hypothesis behavior by humans during discrimination learning. Journal of Experimental Psychology. doi: 10.1037/h0023006

Levine, W. H., \& Hagaman, J. a. (2008). Negated concepts interfere with anaphor resolution. Intercultural Pragmatics, 5(4), 471-500. doi: 10.1515/iprg.2008.023

Lüdtke, J., Friedrich, C. K., De Filippis, M., \& Kaup, B. (2008). Event-related potential correlates of negation in a sentence-picture verification paradigm. Journal of Cognitive Neuroscience, 20(8), 1355-1370. doi: 10.1162/jocn.2008.20093

Lüdtke, J., \& Kaup, B. (2006). Context effects when reading negative and affirmative sentences. In R. Sun \& N. Miyake (Eds.), Proceedings of the 28th Annual Conference of the Cognitive Science Society (pp. 1735-1740). Mahwah, NJ: Lawrence Erlbaum Associates, Inc.

MacDonald, M. C., \& Just, M. A. (1989). Changes in activation levels with negation. Journal of Experimental Psychology Learning Memory and Cognition, 15(4), 633642. doi: 10.1037//0278-7393.15.4.633 
Miller, G. A. (1962). Some psychological studies of grammar. American Psychologist, 17(11), 748-762. doi: dx.doi.org/10.1037/h0044708

Nieuwland, M. S., \& Kuperberg, G. R. (2008). When the truth is not too hard to handle: an event-related potential study on the pragmatics of negation. Psychological Science: A Journal of the American Psychological Society / APS, 19(12), 1213-8. doi: 10.1111/j.1467-9280.2008.02226.x

Rayner, K., \& Pollatsek, A. (1989). The Psychology of Reading. Hillsdale, NJ: Erlbaum. doi:10.4324/9780203357798

Roberts, C. (2012). Information structure in discourse: Towards an integrated formal theory of pragmatics. Semantics and Pragmatics, 5(6), 1-69. doi: 10.3765/sp.5.6

Smithson, M., \& Verkuilen, J. (2006). A better lemon squeezer? Maximum-likelihood regression with beta-distributed dependent variables. Psychological Methods, 11(1), 54-71. doi: 10.1037/1082-989x.11.1.54

Sperber, D. \& Wilson, D. (1986). Relevance: Communication and Cognition. Oxford: Blackwell. doi: 10.1017/s004740450001318x

Stalnaker, R. C. (1978). Assertion. In Cole, P. (Ed.), Syntax and Semantics, Volume 9, pp. 315-332. AP, New York. doi: 10.1002/9780470758335.ch5

Stivers, T., Enfield, N. J., Brown, P., Englert, C., Hayashi, M., Heinemann, T., Hoymann, G., Rossano, F., de Ruiter, J., Yoon, K. \& Levinson, S. C. (2009). Universals and cultural variation in turn-taking in conversation. Proceedings of the National Academy of Sciences, 106(26), 10587-10592. doi:

10.1073/pnas.0903616106

Tanenhaus, M. K., Spivey-Knowlton, M., Eberhard, K., \& Sedivy, J. (1995). Integration of visual and linguistic information in spoken language comprehension. Science, 268(5217), 1632-1634. doi: 10.1126/science.7777863 
Tettamanti, M., Manenti, R., Della Rosa, P. A., Falini, A., Perani, D., Cappa, S. F., \& Moro, A. (2008). Negation in the brain: Modulating action representations. Neuroimage, 43(2), 358-367. doi: j.neuroimage.2008.08.004

Tian, Y., Breheny, R., \& Ferguson, H. J. (2010). Why we simulate negated information: A dynamic pragmatic account. The Quarterly Journal of Experimental Psychology, 63(12), 2305-2312. doi: 10.1080/17470218.2010.525712

Tian, Y., \& Breheny, R. (2015). Dynamic Pragmatic View of Negation Processing. Negation and Polarity: Experimental Perspectives, 21-43. doi:10.1007/978-3-31917464-8_2

Tomasino, B., Weiss, P. H., \& Fink, G. R. (2010). To move or not to move: imperatives modulate action-related verb processing in the motor system. Neuroscience, 169(1), 246-258. doi: 10.1016/j.neuroscience.2010.04.039

Tomlinson, J. M., Bailey, T. M., \& Bott, L. (2013). Possibly all of that and then some: Scalar implicatures are understood in two steps. Journal of Memory and Language, 69(1), 18-35. doi: 10.1016/j.neuroscience.2010.04.039

Tottie, G. (1991). Negation in English speech and writing: a study in variation. San Diego/ New York: Academic Press. doi: 10.2307/416702

Trabasso, T., \& Rollins, H. (1971). Storage and verification stages in processing concepts. Cognitive Psychology, 2(3), 239-289. doi: 10.1016/00100285(71)90014-4

Van Berkum, J., van den Brink, D., Tesink, C. M. J. Y., Kos, M., \& Hagoort, P. (2008). The neural integration of speaker and message. Journal of Cognitive Neuroscience, 20(4), 580-591. doi: 10.1162/jocn.2008.20054

Wason, P. (1965). The contexts of plausible denial. Journal of Verbal Learning Verbal Behavior Vol, 4(1), 7-11. doi: 10.1016/s0022-5371(65)80060-3 


\section{Footnotes:}

"Positive" and "affirmative" are used interchangeably in this paper. We use "argument of negation" to refer to the semantic, conceptual content of the affirmative counterpart of the sentence containing the negative element, "not".

${ }^{2}$ See Geurts (2010) for a discussion of implicatures derived from presuppositions.

${ }^{3}$ But note that other visual-world studies report a delay in access to implicatures see (Tomlinson et al., 2013).

${ }^{4}$ We didn't use adjectives gap fillers such as "Matt has/hasn't shut the bright new window", because adjectives (bright new) often imply visual qualities, which can be used to identify the target. In addition, adjective-noun phrases are often used contrastively, implying a salient alternative with different qualities. This will make the sentences less felicitous against our visual scenes.

${ }^{5}$ We did not instruct the speaker to insert a post-verb silence. It occurred from natural reading.

${ }^{6}$ Please see the Appendix 2 for plots showing the "raw" probability values, separately for target and competitor.

${ }^{7}$ The log correction is applied since fixation proportions are bounded by 0 and 1 .

${ }^{8}$ It is worth noting that the rejection account proposed by Clark and Chase (1972) and Carpenter and Just (1975) was devised in the period before 'real-time' incremental processing was accepted in psycholinguistic research. 


\section{Appendix 1. Experimental sentences}

All experimental sentences appear in four conditions: simple positive, simple negative, cleft positive and cleft negative. For example:

Simple positive: Anna has closed her mom's umbrella.

Simple negative: Anma hasn't closed her mom's umbrella.

Cleft positive: It is Anna who has closed her mom's umbrella.

Cleft negative: It is Anna hasn't closed her mom's umbrella.

The following list are shown in simple negative format.

Anna hasn't closed her mom's umbrella.

Daniel hasn't emptied his mum's saucepan.

Dave hasn't cleaned his wife's wellies.

Grant hasn't sliced his chef's cucumber.

John hasn't ironed his father's shirt.

John hasn't opened his friend's book.

John hasn't turned off his uncle's TV.

Matt hasn't shut his dad's window.

Mike hasn't folded his wife's scarf.

Aiden hasn't washed his dad's car.

Edward hasn't turned on his friend's tap.

Gavin hasn't opened his son's can.

Jim hasn't opened his friend's padlock. 
Mary hasn't folded her friend's deck chair.

Sophie hasn't closed her sister's drawer.

Tina hasn't emptied her mom's jug.

Tracy hasn't sliced her mom's bread.

Amy hasn't finished her cousin's jigsaw puzzle.

Andrew hasn't lit his auntie's candle.

Ben hasn't broken his friend's pencil.

Bill hasn't tied his son's shoe laces.

Chris hasn't fastened his son's zip.

Dave hasn't peeled his sister's banana.

Emma hasn't rolled up her son's sleeves.

Ian hasn't cooked his sister's spaghetti.

James hasn't blown up his cousin's balloon.

James hasn't inflated his brother's tyre.

Jessica hasn't picked up her husband's phone.

Justin hasn't erased his teacher's blackboard.

Lee hasn't sealed his boss's envelope.

Lilly hasn't cracked her sister's egg.

Linda hasn't iced her auntie's cupcake.

Lucas hasn't turned off his wife's light bulb.

Lucy hasn't framed her sister's photo.

Nathan hasn't emptied his colleague's truck.

Nick hasn't decorated his friend's Christmas tree.

Paul hasn't dried his auntie's tomatoes.

Rita hasn't screwed up her sister's wrapping paper. 
Susan hasn't mended her son's jeans.

Susan hasn't rolled up her friend's yoga mat.

Zoey hasn't cut her sister's cake.

Appendix 2. Figures showing proportions of looks to the target and the competitor separately, for simple and cleft conditions.

Figure Appendix 2a here

Figure Appendix $2 b$ here 


\section{Tables}

Table 1. Design and examples of experimental and filler sentences

\begin{tabular}{|c|c|c|c|c|}
\hline $\begin{array}{l}\text { Exp./ } \\
\text { Filler }\end{array}$ & Condition & Cleft & $\begin{array}{l}\text { Number } \\
\text { of items }\end{array}$ & Example \\
\hline \multirow{4}{*}{ Exp. } & has & & 10 & Anna has closed her mom's umbrella. \\
\hline & hasn't & & 10 & Matt hasn't shut his dad's window. \\
\hline & has & & 10 & It is James who has blown up his cousin's balloon. \\
\hline & hasn't & & 10 & It is Lilly who hasn't cracked her sister's egg. \\
\hline \multirow{7}{*}{ Filler } & & simple & 5 & Bob will chop his father's wood. \\
\hline & & cleft & 5 & Andrew will ride his father's horse. \\
\hline & & simple & 5 & Bill has wrapped the birthday present. \\
\hline & & & & It is Lucy who should have watered her Dad's \\
\hline & have & cleft & 5 & flower. \\
\hline & shouldn't & simple & 10 & Eva shouldn't have scratched her brother's CD. \\
\hline & have & cleft & 10 & It is Betty who shouldn't have cut her friend's rope. \\
\hline
\end{tabular}


Table 2. Average word lengths in milliseconds for key regions

\begin{tabular}{cccccc}
$\begin{array}{c}\text { Average word } \\
\text { length in ms }\end{array}$ & verb & $\begin{array}{c}\text { post-verb } \\
\text { silence }\end{array}$ & his/her & someone's & noun \\
\hline $\begin{array}{l}\text { Simple } \\
\text { Has }\end{array}$ & 504 & 222 & 169 & 424 & 546 \\
$\quad$ Hasn't & 501 & 151 & 169 & 441 & 577 \\
Cleft & & 10 & 174 & 420 & 549 \\
Has & 438 & 38 & 175 & 419 & 578 \\
Hasn't & 467 & & & & \\
\hline
\end{tabular}


Table 3. Proportion of looks to target, competitor, person and distractors for all conditions in key regions. The numbers for distractors correspond to the summed percentage of looks to both distractors.

\begin{tabular}{|c|c|c|c|c|c|}
\hline \multicolumn{2}{|c|}{ Proportion of looks by condition } & \multirow{2}{*}{$\begin{array}{l}\text { verb } \\
0.13\end{array}$} & \multirow{2}{*}{$\begin{array}{l}{[\mathrm{SIL}] \&} \\
\text { "his/her" } \\
0.25\end{array}$} & \multirow{2}{*}{$\begin{array}{l}\text { someone's } \\
0.32\end{array}$} & \multirow{2}{*}{$\begin{array}{l}\text { noun } \\
0.35\end{array}$} \\
\hline & target & & & & \\
\hline & competitor & 0.13 & 0.13 & 0.13 & 0.16 \\
\hline \multirow[t]{5}{*}{ Simple Positive } & target \& competitor Total & 0.26 & 0.38 & 0.45 & 0.51 \\
\hline & person & 0.29 & 0.2 & 0.16 & 0.14 \\
\hline & distractors & 0.25 & 0.24 & 0.23 & 0.16 \\
\hline & target & 0.15 & 0.21 & 0.27 & 0.32 \\
\hline & competitor & 0.11 & 0.21 & 0.2 & 0.2 \\
\hline \multicolumn{2}{|c|}{ Simple Negative target \& competitor Total } & 0.26 & 0.42 & 0.47 & 0.52 \\
\hline & person & 0.28 & 0.19 & 0.18 & 0.14 \\
\hline & distractors & 0.25 & 0.21 & 0.18 & 0.16 \\
\hline \multirow{5}{*}{ Cleft Positive } & target & 0.13 & 0.14 & 0.24 & 0.38 \\
\hline & competitor & 0.12 & 0.14 & 0.17 & 0.16 \\
\hline & target \& competitor Total & 0.25 & 0.29 & 0.41 & 0.54 \\
\hline & person & 0.3 & 0.28 & 0.23 & 0.14 \\
\hline & distractors & 0.29 & 0.25 & 0.2 & 0.15 \\
\hline \multirow{5}{*}{ Cleft Negative } & target & 0.14 & 0.2 & 0.26 & 0.31 \\
\hline & competitor & 0.15 & 0.2 & 0.19 & 0.19 \\
\hline & target \& competitor Total & 0.29 & 0.4 & 0.45 & 0.5 \\
\hline & person & 0.24 & 0.23 & 0.17 & 0.13 \\
\hline & distractors & 0.28 & 0.2 & 0.19 & 0.18 \\
\hline
\end{tabular}


Table 4. Paired sample t-tests on target advantage scores between positive and negative. Asterisks in this table and the rest of the paper indicate levels of significance based on $p$ values corrected for multiple comparisons. $*$ for $\mathrm{p}<0.05, * *$ for $\mathrm{p}<0.01$, and $* * *$ for $\mathrm{p}<0.001$.

\begin{tabular}{lcccccccc} 
& \multicolumn{3}{c}{ By subject } & & \multicolumn{3}{c}{ By item } \\
\cline { 2 - 4 } \cline { 6 - 8 } Simple & $\mathrm{df}$ & $\mathrm{t}_{1}$ & $\mathrm{p}$ (2-tailed) & & $\mathrm{df}$ & $\mathrm{t}_{2}$ & $\mathrm{p}$ (2-tailed) \\
verb & 35 & 0.89 & 0.38 & & 38 & 0.75 & 0.46 \\
SIL\&his & 35 & $3.20^{* *}$ & 0.003 & & 38 & $2.195^{*}$ & 0.03 \\
someone's & 35 & $4.52^{* * *}$ & $6.8 \mathrm{E}-05$ & & 38 & $2.995^{* *}$ & 0.005 \\
noun & 35 & $3.26^{* *}$ & 0.003 & & 38 & $2.435^{*}$ & 0.02 \\
Cleft & & & & & & & & \\
verb & 35 & 0.71 & 0.48 & & 38 & 0.53 & 0.60 \\
SIL\&his & 35 & 0.40 & 0.69 & & 38 & 0.026 & 0.98 \\
someone's & 35 & 0.21 & 0.84 & & 38 & 1.226 & 0.23 \\
noun & 35 & $3.22^{* *}$ & 0.003 & 38 & 1.738 & 0.09 \\
\hline
\end{tabular}


Table 5. One sample t-test comparing target advantage scores with zero on all conditions.

By subject

Simple

A verb

SIL \& his

$35 \quad 0.28$

0.78

$6.4 \mathrm{E}-05$

someone's

noun

35

$\mathrm{N}$

verb

SIL \& his

35

0.18

0.364

0.003

0.92

$3.15^{* *}$

3.0E-08

someone's $\quad 35$

35

38

$6.20 * * *$

3.0E-07

Cleft

A verb

$35 \quad 0.16$

0.87

38

0.14

0.89

SIL \& his

35

0.19

0.85

38

0.5

0.61

someone's $\quad 35 \quad 2.63 *$

0.01

38

$2.49 *$

0.02

N

$355.82 * * *$

1.4E-06

38

$6.17 * * *$

3.3E-07

verb

$35 \quad 1.50$

0.14

38

0.68

0.50

SIL \& his

$\begin{array}{ll}35 & 0.37\end{array}$

0.715

38

0.61

0.54

someone's

$353.03^{* *}$

0.005

38

1.61

0.12

noun

$35 \quad 3.20^{* *} \quad 0.003$

38

$3.16^{* *}$

0.003 
Table 6. One sample t-test comparing target advantage scores with zero in 100ms time slices from the offset of verb

\begin{tabular}{|c|c|c|c|c|c|c|c|c|c|}
\hline \multirow[b]{2}{*}{ Simple } & \multirow{2}{*}{$\begin{array}{l}\text { 100ms } \\
\text { slice } \\
\text { post } \\
\text { verb }\end{array}$} & \multicolumn{3}{|c|}{ By subject } & \multicolumn{5}{|c|}{ By item } \\
\hline & & $\mathrm{df}$ & $\mathrm{t}_{1}$ & $\begin{array}{l}\mathrm{p}(2- \\
\text { tailed })\end{array}$ & $\begin{array}{l}\text { P (Šidák } \\
\text { corrected) }\end{array}$ & $\mathrm{df}$ & $\mathrm{t}_{2}$ & $\begin{array}{l}\mathrm{p}(2- \\
\text { tailed })\end{array}$ & $\begin{array}{l}\text { P (Šidák } \\
\text { corrected) }\end{array}$ \\
\hline \multirow[t]{10}{*}{$\mathrm{A}$} & 1 & 35 & 1.10 & 0.28 & 1 & 38 & 0.75 & 0.46 & 1 \\
\hline & 2 & 35 & $3.17 *$ & $3.1 \mathrm{E}-03$ & 0.03 & 38 & 2.69 & 0.01 & 0.10 \\
\hline & 3 & 35 & $5.81 * * *$ & $1.4 \mathrm{E}-06$ & $1.3 \mathrm{E}-05$ & 38 & $5.07 * * *$ & $1.0 \mathrm{E}-05$ & $1.0 \mathrm{E}-04$ \\
\hline & 4 & 35 & $5.87 * * *$ & $1.1 \mathrm{E}-06$ & $1.1 \mathrm{E}-05$ & 38 & $5.26 * * *$ & $5.5 \mathrm{E}-06$ & $5.3 \mathrm{E}-05$ \\
\hline & 5 & 35 & $5.63 * * *$ & 2.4E-06 & $2.3 \mathrm{E}-05$ & 38 & $5.24 * * *$ & $5.8 \mathrm{E}-06$ & $5.7 \mathrm{E}-05$ \\
\hline & 6 & 35 & $5.88 * * *$ & $1.1 \mathrm{E}-06$ & $1.1 \mathrm{E}-05$ & 38 & $5.04 * * *$ & 1.1E-05 & $1.1 \mathrm{E}-04$ \\
\hline & 7 & 35 & $5.69 * * *$ & $2.0 \mathrm{E}-06$ & $1.9 \mathrm{E}-05$ & 38 & $5.94 * * *$ & $6.8 \mathrm{E}-07$ & $6.7 \mathrm{E}-06$ \\
\hline & 8 & 35 & $5.89 * * *$ & $1.1 \mathrm{E}-06$ & $1.1 \mathrm{E}-05$ & 38 & $6.07 * * *$ & 4.6E-07 & 4.5E-06 \\
\hline & 9 & 35 & $5.33 * * *$ & $5.9 \mathrm{E}-06$ & $5.8 \mathrm{E}-05$ & 38 & $5.07 * * *$ & 1.1E-05 & $1.0 \mathrm{E}-04$ \\
\hline & 10 & 35 & $5.41 * * *$ & 4.6E-06 & 4.5E-05 & 38 & $5.38 * * *$ & 4.0E-06 & $3.9 \mathrm{E}-05$ \\
\hline \multirow[t]{10}{*}{$\mathrm{N}$} & 1 & 35 & 0.63 & 0.54 & 1 & 38 & 0.13 & 0.90 & 1 \\
\hline & 2 & 35 & 0.58 & 0.57 & 1 & 38 & 1.16 & 0.25 & 1 \\
\hline & 3 & 35 & 0.98 & 0.33 & 1 & 38 & 0.95 & 0.35 & 1 \\
\hline & 4 & 35 & 0.72 & 0.47 & 1 & 38 & 0.65 & 0.52 & 1 \\
\hline & 5 & 35 & 0.33 & 0.74 & 1 & 38 & 0.49 & 0.62 & 1 \\
\hline & 6 & 35 & 1.07 & 0.29 & 1 & 38 & 1.79 & 0.08 & 0.78 \\
\hline & 7 & 35 & 1.03 & 0.31 & 1 & 38 & 2.04 & 0.05 & 0.49 \\
\hline & 8 & 35 & 1.52 & 0.14 & 1 & 38 & 2.78 & 0.01 & 0.10 \\
\hline & 9 & 35 & 2.03 & 0.05 & 0.49 & 38 & 2.44 & 0.02 & 0.2 \\
\hline & 10 & 35 & 2.65 & 0.01 & 0.10 & 38 & 2.85 & 0.007 & 0.07 \\
\hline
\end{tabular}

Cleft

$\begin{array}{cccccccccc}\mathrm{A} & 1 & 35 & 0.61 & 0.54 & 1 & 38 & 0.75 & 0.46 & 1 \\ & 2 & 35 & 0.84 & 0.40 & 1 & 38 & 0.05 & 0.96 & 1 \\ 3 & 35 & 1.10 & 0.28 & 1 & 38 & 0.73 & 0.47 & 1 \\ 4 & 35 & 1.09 & 0.28 & 1 & 38 & 0.94 & 0.35 & 1 \\ 5 & 35 & 2.87 & 0.007 & 0.07 & 38 & 2.80 & 8.0 \mathrm{E}-03 & 0.08 \\ 6 & 35 & 4.07 * * * & 2.0 \mathrm{E}-04 & 0.002 & 38 & 3.48^{*} & 1.0 \mathrm{E}-03 & 0.01 \\ 7 & 35 & 5.31^{* * *} & 6.2 \mathrm{E}-06 & 6.1 \mathrm{E}-05 & 38 & 4.33^{* *} & 1.1 \mathrm{E}-04 & 0.001 \\ 8 & 35 & 5.57^{* * *} & 2.9 \mathrm{E}-06 & 2.8 \mathrm{E}-05 & 38 & 5.15^{* * *} & 8.3 \mathrm{E}-06 & 8.1 \mathrm{E}-05 \\ 9 & 35 & 6.17^{* * *} & 4.6 \mathrm{E}-07 & 4.5 \mathrm{E}-06 & 38 & 5.41^{* * *} & 3.7 \mathrm{E}-06 & 3.6 \mathrm{E}-05\end{array}$




\begin{tabular}{cccccccccc} 
& 10 & 35 & $5.96^{* * *}$ & $8.8 \mathrm{E}-07$ & $8.6 \mathrm{E}-06$ & 38 & $4.86^{* * *}$ & $2.0 \mathrm{E}-05$ & $2.0 \mathrm{E}-04$ \\
\hline $\mathrm{N}$ & 1 & 35 & 0.30 & 0.77 & 1 & 38 & 0.24 & 0.81 & 1 \\
& 2 & 35 & 0.45 & 0.65 & 1 & 38 & 1.07 & 0.29 & 1 \\
& 3 & 35 & 1.53 & 0.14 & 1 & 38 & -0.04 & 0.97 & 1 \\
& 4 & 35 & 1.85 & 0.07 & 0.68 & 38 & 0.48 & 0.63 & 1 \\
& 5 & 35 & 2.43 & 0.02 & 0.2 & 38 & 1.43 & 0.16 & 1 \\
6 & 35 & $3.52^{* *}$ & $1.2 \mathrm{E}-03$ & 0.01 & 38 & 2.23 & 0.03 & 0.29 \\
7 & 35 & $3.81 * *$ & $5.3 \mathrm{E}-04$ & 0.005 & 38 & 2.08 & 0.04 & 0.39 \\
& 8 & 35 & 2.37 & 0.02 & 0.2 & 38 & 2.32 & 0.03 & 0.29 \\
9 & 35 & 2.41 & 0.02 & 0.2 & 38 & $3.25 *$ & 0.002 & 0.02 \\
10 & 35 & 1.29 & 0.21 & 1 & 38 & 2.62 & 0.01 & 0.098 \\
\hline
\end{tabular}

Figure captions:

Figure 1. Target advantage scores for positive and negative conditions - simple

Figure 2. Target advantage scores for positive and negative conditions - cleft

Figure 3. Average target advantage scores in 100 time slices from the offset of verb Simple.

Figure 4. Average target advantage scores in 100 time slices from the offset of verb Cleft.

Figure Appendix 2a. Proportions of looks to the target and the competitor_simple Condition

Figure Appendix 2b. Proportions of looks to the target and the competitor_cleft condition 